\title{
Non-Cooperative Resource Competition Game by Virtual Referee in Multi-Cell OFDMA Networks
}

\author{
Zhu Han, Zhu Ji, and K. J. Ray Liu
}

\begin{abstract}
In this paper, a distributive non-cooperative game is proposed to perform sub-channel assignment, adaptive modulation, and power control for multi-cell multi-user Orthogonal Frequency Division Multiplexing Access (OFDMA) networks. Each individual user's goal is to minimize his/her own transmitted power in a distributed manner under the constraints that the desirable rate is achieved and the transmitted power is bounded. The pure non-cooperative game may result in non-convergence or some undesirable Nash Equilibriums with low system and individual performances. To enhance the performances, a virtual referee is introduced to the networks and is in charge of monitoring and improving the outcome of non-cooperative competition for resources among the distributed users. If the game outcome is not desirable, either the required transmission rates should be reduced or some users should be prevented from using some radio resources such as sub-channels, so that the rest of users can share the limited resources more efficiently. Moreover, it can be shown that the introduction of the virtual referee does not increase the complexity of the networks. From the simulation results in a two-cell case, the proposed scheme reduces the transmitted power by $80 \%$ and $25 \%$ compared with the fixed channel assignment algorithm and the iterative water-filling algorithm in the literature, respectively. The achievable rate can be improved by $10 \%$. In a multi-cell case, the proposed scheme can have up to $40 \%$ power reduction compared with the iterative water-filling algorithm when the co-channel interferences are severe.
\end{abstract}

Index Terms-Resource allocation, power control, spectrum allocation, game theory, and OFDMA.

\section{INTRODUCTION}

$\mathbf{O}$ RTHOGONAL Frequency Division Multiplexing Access (OFDMA) prevails in the proposed future wireless network standards like 4G cellular networks, Wireless MAN, Wireless LAN, and Ultra Wide Band networks. OFDMA creates a robust multiple access scheme to deal with the impairments and uncertainties of wireless channels. To fully take advantages of OFDMA, resource allocation should be employed to explore the time, frequency, and multi-user diversities. Resource allocation for OFDMA networks has three major tasks: sub-channel assignment, rate allocation, and power control. Sub-channel assignment enables the efficient usage of the sub-channels according to the channel conditions, and limits the co-channel interferences. Rate adaptation such as the adaptive modulation technique provides good

Manuscript received July 1, 2006; revised February 15, 2007. This work is partially supported by MURI AFOSR F496200210217.

Zhu Han is with the Electrical and Computer Engineering Department, Boise State University, ID (e-mail: hanzhu22@gmail.com).

K. J. Ray Liu is with the Electrical and Computer Engineering Department, and Institute for Systems Research, University of Maryland, College Park, MD (e-mail: kjrliu@umd.edu).

Zhu Ji is with QUALCOMM, San Diego, CA (e-mail: zhuji@umd.edu).

Digital Object Identifier 10.1109/JSAC.2007.070803. potential to vary the number of transmitted bits for each sub-channel according to the instantaneous sub-channel link quality, while maintaining an acceptable Bit Error Rate (BER). Power control constantly adjusts the transmission power so as to maintain the link quality. All these tasks pose some significant challenges on the design of resource allocation schemes for OFDMA systems.

In a multi-cell OFDMA system, the resource allocation problem becomes more complicated, because the distributive topology of the system requires distributive implementations. Moreover, since the co-channel interferences are affected by the sub-channel assignment, the corresponding rate adaptation and power control, any change of resource allocation in a specific cell will affect the performances of other nearby cells. In the literature, when the sub-channel assignment is fixed, several iterative water-filling methods are proposed in [1]-[4] to maximize the throughput with power constraints. However, if the sub-channel assignment to the users is not predetermined, all possible combinations of the co-channel users need be checked to determine the best resource allocation, which highly increases the complexity of resource allocation. In [5], the authors present a heuristic distributed algorithm that is executed independently by each base station. The algorithm is based on iterative water-filling by removing the sub-channels when the signal to interferences and noise ratio (SINR) is lower than a threshold. In [6], a semi-distributed dynamic resource allocation scheme is proposed for downlink multi-cell OFDMA networks. In [7], channel allocations with adaptive modulation and power control in a multi-cell system are studied for generic multiple access schemes with orthogonal channels.

In the multi-cell case, it is difficult for an individual user to know the channel conditions of the other users in the other cells. Thus the users in different cells cannot cooperate with each other. They act selfishly to maximize their own performances in a distributive fashion. Such a fact motivates us to adopt the game theory [8]. The resource allocation can be modelled as a non-cooperative game that deals largely with how rational and intelligent individuals interact with each other in an effort to achieve their own goals. In this game, each mobile user is self-interested and trying to optimize his/her utility function, where the utility function represents the user's performance and controls the outcomes of the game.

In the literature, different types of game approaches have been introduced to several areas of wireless communications. The non-cooperation game theory was studied in [9] for power control problems, where the pricing technique was used to achieve Pareto optimality. Pricing Anarchy is discussed in 
details in [10] for routing problem. In [11], forward link power allocation and admission control policies for voice users in a code-division multiple access (CDMA) wireless network are investigated. In [12], [13], the reputation-based game approaches were proposed to encourage packet-forwarding among users. In [14], the authors proposed a non-cooperative approach for encouraging collaboration in MONET. In [15], the mechanism design scheme for truthful revelation is constructed for differentiated service multicast. In regard to the cooperation game theory, a framework was described in [16] for bandwidth allocation of elastic services in high-speed networks. In [17], a cooperative game approach named Nash Bargaining Solution was studied in the scenario of power, rate, and sub-channel allocation for single-cell OFDMA systems to have a fair and efficient performance. The bound of performance ratio of the worst case over optimal case for spectrum sharing games is developed in [18]. In [19], the ideas in [17] and [18] are combined for the spectrum allocation. In [20] and [21], a repeated game approach and a self learning repeated game approach are proposed for packet forwarding networks to ensure cooperation and study how to cooperate among the distributed and greedy nodes. In [22], the game theory approach is proposed to combine with the idea in [5] for resource allocation problems in multicell OFDMA systems. A general tutorial for non-cooperative game over wireless networks is given in [23].

By developing a non-cooperative game theory approach from the individual point of view, each individual user minimizes his/her own transmitted power, under the rate and power constraints. From the system point of view, the overall transmitted power of multi-cell OFDMA systems is minimized under the constraints mentioned above. However, the outcomes of individual optimization might not always be as good as those of system optimization. By exploring a two-user twosub-channel example, we find the following facts: If the cochannel interferences are small, users can share the subchannels for transmission. In this case, by carefully designing the utility function, the distributive non-cooperative game will be balanced at a unique Nash equilibrium point (NEP) which is also optimal from the system point of view. If the cochannel interferences become severe on some sub-channels, NEP may not be optimal for system optimization, and there might be multiple NEPs and multiple local optima. In order to improve the undesired game outcomes, some users with bad channel conditions or causing large interferences to others must be prevented from using these sub-channels, or these users' required transmission rates should be reduced. By doing so, the rest of the co-channel users can share the corresponding sub-channels more efficiently.

Based on these observations, we introduce a virtual referee which is similar to the function of referee in basketball or soccer games in daily life. If some player behaves badly or injures others during the game, a referee can force him/her out of the game. Here in the multicell OFDMA networks, a referee is introduced to the networks and is in charge of monitoring and improving the outcome of non-cooperative competition for resources among the distributed users. If the outcome is desirable, the referee does nothing. Otherwise, the referee will mandatorily change the rule of the non-cooperative game such as removing users from using sub-channels or reducing the required transmission rates. By doing this, the non-cooperative game can be balanced at some better Nash equilibriums so that the limited resources can be shared more efficiently. There are two differences of the proposed problem formulation from the iterative water-filling [2] in the literature. First, we optimize which user can transmit on some specific sub-channel, so that we have one more degree of freedom to optimize the resource usage. Second, the individual optimization problem is the dual problem of the iterative water-filling and the system is non-linear and non-convex. Moreover, the virtual referee is shown to be easy to implement in practice. The simulation results show the proposed scheme can reduce the overall transmitted power greatly while achieving higher transmission rates, compared to the fixed channel assignment algorithm and the iterative water-filling algorithm.

The rest of this paper is organized as follows: In Section II, we illustrate the system model. Then we formulate the optimization problems over the multicell multiuser OFDMA network for system optimization and individual optimization, respectively. In Section III, a virtual referee is introduced to improve the distributive non-cooperative game, based on the discussion for the two-user two-subchannel case. The game properties of the proposed game are studied and practical implementation issues are discussed. In Section IV, we have the simulation studies. In Section V, the conclusions are drawn.

\section{System Model And Problem Formulations}

In this section, we first provide the system model. Then we formulate the system optimization problem and propose the individual non-cooperative game. Finally, the feasibility region for resource allocation is analyzed.

\section{A. System Model}

We consider an OFDMA network with $K$ co-channel links that exist in the distinct cells and share the same spectrum. The co-channel links cause interference among each other. Each link consists of a mobile user and its assigned base station. Assume coherent detection is possible so that it is sufficient to model this multiuser system by an equivalent baseband model. The total number of OFDM sub-channels is $L$, and each sub-channel can carry different data. For the uplink case, the sampled signal on the $l^{\text {th }}$ sub-channel of the $i^{\text {th }}$ user can be expressed as:

$$
x_{i}^{l}(n)=\sum_{j=l-1}^{l+1} \sum_{k=1}^{K} \sqrt{\theta_{k i}^{j l} P_{k}^{j} G_{k i}^{j}} s_{k}^{j}(n)+n_{i}^{l}(n)
$$

where $P_{k}^{l}$ and $G_{k i}^{l}$ is the transmitted power and propagation loss from the $k^{t h}$ user to the $i^{t h}$ base station in the $l^{\text {th }}$ subchannel, respectively, $\theta_{k i}^{j l}$ is the carrier correlation between the $k^{t h}$ user's transmitter's $j^{\text {th }}$ sub-channel and the $i^{\text {th }}$ user's receiver's $l^{\text {th }}$ sub-channel, $s_{k}^{l}$ is message symbol from the $k^{t h}$ user to the $i^{t h}$ base station at time $n$, and $n_{i}^{l}(n)$ is the sampled thermal noise. We assume that the channels change slowly and the channel gain is stable within each frame. If the network is synchronized $^{1}, \theta_{k i}^{j l}=1$, if $j=l ; \theta_{k i}^{j l}=0$, otherwise. Without

\footnotetext{
${ }^{1}$ All users transmit relatively at the same time within the accuracy of guardband.
} 
loss of generality, we assume the thermal noise for each user and each sub-channel is the same, i.e., $N_{i}^{l}=E\left(\left\|n_{i}^{l}\right\|^{2}\right)=N_{0}$. The $i^{t h}$ user's SINR at sub-channel $l$ can be expressed as:

$$
\Gamma_{i}^{l}=\frac{P_{i}^{l} G_{i i}^{l}}{\sum_{k \neq i} P_{k}^{l} G_{k i}^{l}+N_{0}} .
$$

Notice that for the downlink synchronized multi-cell case, the SINR expression is similar to (2) by replacing $G_{k i}^{l}$ with $G_{i k}^{l}$. So all the deduction and analysis in the rest of the paper can be employed to the downlink case in a similar way.

Rate adaptation such as adaptive modulation provides each sub-channel with the ability to match the effective bit rates, according to the interference and channel conditions. Quadrature Amplitude Modulation (QAM) is a modulation method with high spectrum efficiency. Without loss of generality, we assume the output of the different adaptive modulation constellation has unit power. In [24], [25], given a desirable rate $r_{i}^{l}$ of MQAM, the BER of the $l^{t h}$ sub-channel of the $i^{t h}$ user can be approximated as a function of the received SINR $\Gamma_{i}^{l}$ by:

$$
\mathrm{BER}_{i}^{l} \approx c_{1} e^{-c_{2} \frac{\Gamma_{i}^{l}}{2^{r_{i}^{l}}-1}}
$$

where $c_{1} \approx 0.2$ and $c_{2} \approx 1.5$ with a small $\mathrm{BER}_{i}^{l}$. Further, given a specific desirable $\mathrm{BER}_{i}^{l}$, by rearranging (3), the $i^{t h}$ user's transmission rate on the $l^{\text {th }}$ sub-channel can be given by:

$$
r_{i}^{l}=W \log _{2}\left(1+c_{3}^{i} \Gamma_{i}^{l}\right) \text { bit/symbol }
$$

where $W$ is the bandwidth, $c_{3}^{i}=-\frac{c_{2}^{i}}{\ln \left(\mathrm{BER}_{i}^{l} / c_{1}^{i}\right)}$, and $\Gamma_{i}^{l}$ is SINR. In this paper, for simplicity, we assume all subchannels and users have the same BER requirement, i.e., $\mathrm{BER}_{i}^{l}=\mathrm{BER}, \forall i, l$.

Each user requires the rate $R_{i}$ and allocates its rate into $L$ sub-channels, i.e., $\sum_{l=1}^{L} r_{i}^{l}=R_{i}, \forall i$. Each user's transmitted power is bounded by $P_{\text {max }}^{i}$, i.e., $\sum_{l=1}^{L} P_{i}^{l} \leq P_{\max }^{i}, \forall i$. Without loss of generality, in this paper we assume all users have the same maximal power constraint $P_{\max }$. Define the $K \times L$ channel assignment matrix $\mathbf{A}$ as

$$
[\mathbf{A}]_{i l}= \begin{cases}1, & \text { if } r_{i}^{l}>0, \text { i.e., } P_{i}^{l}>0 \\ 0, & \text { otherwise. }\end{cases}
$$

Define rate allocation matrix as $[\mathbf{r}]_{i l}=r_{i}^{l}$.

\section{B. System Optimization}

From the system optimization point of view, the objective is to minimize the overall transmitted power under the rate and power constraints, by adjusting the rate allocation over different sub-channels for different users, i.e.,

$$
\min _{\mathbf{A}, \mathbf{r}} f(\mathbf{r})=\sum_{i=1}^{K} \sum_{l=1}^{L} P_{i}^{l}
$$

$$
\text { s.t. }\left\{\begin{array}{l}
\text { Rate Requirement: } \sum_{l=1}^{L} r_{i}^{l}-R_{i}=0, \forall i, \\
\text { Maximum Power Contraint: } \\
\quad \sum_{l=1}^{L} P_{i}^{l}-P_{\max } \leq 0, \forall i, \\
\text { Non-negative Contraint: } r_{i}^{l}, P_{i}^{l} \geq 0, \forall i, l,
\end{array}\right.
$$

where $P_{i}^{l}$ is a function of $\mathbf{A}$ and $\mathbf{r}$. Notice that $\mathbf{A}$ is an indication matrix on whether or not the components of $\mathbf{r}$ are positive or zero. Since the components of $\mathbf{A}$ are integers, the problem in (6) is a generalized knapsack problem [33] and is a high order $N P$ hard optimization problem. Moreover, in order to solve (6) by centralized constrained optimization methods, all information about channels is required. This causes impractically large amount of communication overheads between the distinct cells. This motivates us to develop a distributed algorithm using the game theory approach, where only location information is necessary for optimization.

\section{Pure Non-cooperative Game for Individual User}

In this subsection, a pure non-cooperative game is constructed. First, we give some basic definitions about noncooperative games. A game can be defined as each user adjusts his/her strategy (like rates) to optimize his/her own utility to compete with others. Strategy and utility can be defined as:

Definition 1: A strategy $r$ is a complete contingent plan, or a decision rule, that defines the action an agent will select in every distinguishable state $\Omega$.

Definition 2: In any game, utility $u$ represents the motivations of players. A utility function for a given player assigns a number for every possible outcome of the game with the property that a higher (or lower) number implies that the outcome is more preferred.

To analyze the outcome of the game, Nash Equilibrium is a well-known concept, which states that in the equilibrium every agent will select a utility-maximizing strategy given the strategies of every other agent.

Definition 3: Define a strategy rate vector $\mathbf{r}=\left[r_{1} \ldots r_{K}\right]$ and define the rate vector of the $i^{t h}$ player's opponents as $\mathbf{r}_{i}^{-1}=\left[\begin{array}{lll}r_{1} \ldots r_{i-1} & r_{i+1} \ldots r_{K}\end{array}\right]$, where $K$ is number of users and $r_{i}$ is the $i^{t h}$ user's rate. $u_{i}$ is the $i^{t h}$ user's utility. Nash Equilibrium Point $\mathbf{r}^{*}$ is defined as:

$$
u_{i}\left(r_{i}^{*}, \mathbf{r}_{i}^{-1}\right) \geq u_{i}\left(\tilde{r}_{i}, \mathbf{r}_{i}^{-1}\right), \forall i, \forall \tilde{r}_{i} \in \Omega, \mathbf{r}_{i}^{-1} \in \Omega^{K-1} .
$$

i.e., given the other users' resource allocations, no user can increase his/her utility unilaterally by changing his/her own resource allocation.

In the multi-cell system, each distributed user wants to minimize its transmitted power by allocating its rate into the different sub-channels, regardless other users in a distributed way. Define rate vector $\mathbf{r}_{i}=\left[r_{i}^{1} \ldots r_{i}^{L}\right]^{T}$. For individual optimization, the non-cooperative game can be written as:

Non-Cooperative Game: $\min _{\mathbf{r}_{i} \in \Omega} u_{i}=\sum_{l=1}^{L} P_{i}^{l}$, s.t. $\sum_{l=1}^{L} r_{i}^{l}=R_{i}$,

where $u_{i}$ is the utility function defined as the $i^{\text {th }}$ user's transmitting power. Notice that here the utility is better when it is smaller. If the overall power for each user is larger than $P_{\max }$, we will develop another approach in the next session. In the following analysis of this subsection, we assume that the power constraint can be satisfied.

If the interferences from others are fixed, we can consider the interferences as noise. So the problem in (8) is a waterfilling problem [27]. Define

$$
I_{i}^{l}=\frac{1}{c_{3}^{i} \Gamma_{i}^{l}}=\frac{\sum_{k \neq i} P_{k}^{l} G_{k i}^{l}+N_{0}}{c_{3}^{i} G_{i i}^{l}},
$$


the solution is

$$
P_{i}^{l}=\left(\mu_{i}-I_{i}^{l}\right)^{+} \text {and } r_{i}^{l}=\log _{2}\left(1+\frac{P_{i}^{l}}{I_{i}^{l}}\right)
$$

where $y^{+}=\max (y, 0) \cdot \mu_{i}$ is solved by bisection search of

$$
\sum_{l=1}^{L} \log _{2}\left(1+\frac{\left(\mu_{i}-I_{i}^{l}\right)^{+}}{I_{i}^{l}}\right)=R_{i} .
$$

The interferences are constantly changed by the others' game strategies. The above water-filling solution provides the best utility given the other users' resource allocation. So the resulting game outcome is a Nash equilibrium. The above problem formulation is a dual problem [26] of the iterative water-filling [2] in the literature.

\section{System Feasibility Region}

In this subsection, we give a necessary condition for the feasibility of the problems in (6) and (8). In order to ensure the desirable BER, for every sub-channel, every user should have SINR no less than a required SINR $\gamma_{i}^{l}$, i.e., $\Gamma_{i}^{l} \geq \gamma_{i}^{l}, \forall i, l$. Rewrite these inequalities in matrix form, we have

$$
\left(\mathbf{I}-\mathbf{D}^{l} \mathbf{F}^{l}\right) \mathbf{P}^{l} \geq \mathbf{v}^{l}, \forall l,
$$

where $\mathbf{I}$ is a $K \times K$ identity matrix, $\mathbf{D}^{l}=\operatorname{diag}\left\{\gamma_{1}^{l}, \ldots, \gamma_{K}^{l}\right\}$,

$$
\left[\mathbf{F}_{i j}^{l}\right]= \begin{cases}0 & \text { if } j=i \\ \frac{G_{j i}^{l}}{G_{i i}^{l}} & \text { if } j \neq i\end{cases}
$$

and $\mathbf{v}^{l}=\left[v_{1}^{l}, \ldots, v_{K}^{l}\right]^{\prime}$ with $v_{i}^{l}=\frac{N_{0} \gamma_{i}^{l}}{G_{i i}}$. By Perron-Frobenius theorem [30], there exists a non-negative power allocation if and only if the maximum eigenvalue of $\mathbf{D}^{l} \mathbf{F}^{l}$, i.e. spectrum radius $\rho\left(\mathbf{D}^{l} \mathbf{F}^{l}\right)$, is inside the unit circle. Moreover, the optimal power solution is

$$
\mathbf{P}^{l}= \begin{cases}\left(\mathbf{I}-\mathbf{D}^{l} \mathbf{F}^{l}\right)^{-1} \mathbf{v}^{l}, & \left|\rho\left(\mathbf{D}^{l} \mathbf{F}^{l}\right)\right|<1 \\ +\infty, & \text { otherwise }\end{cases}
$$

The system feasibility region $\Omega$ is defined as the supporting domain where there exist non-infinity solutions and power constraint in (6) is satisfied. The condition for (13) to have finite solutions is a necessary condition for existence of a feasible range $\Omega$, i.e., for any feasible solution $\mathbf{r}$, we have $\mathbf{r} \in \Omega$ with $\rho\left(\mathbf{D}^{l} \mathbf{F}^{l}\right)<1, \forall l$.

\section{IMPROVEMENT OF NON-COOPERATIVE GAME BY VIRTUAL REFEREE}

In this section, a two-user two-sub-channel example is first given to show the insights. Then based on the observations of the example, the Lemmas of the NEP are analyzed. Next, with the analytical results, an iterative resource allocation algorithm with a virtual referee is proposal. Finally, the practical implementation considerations are discussed.

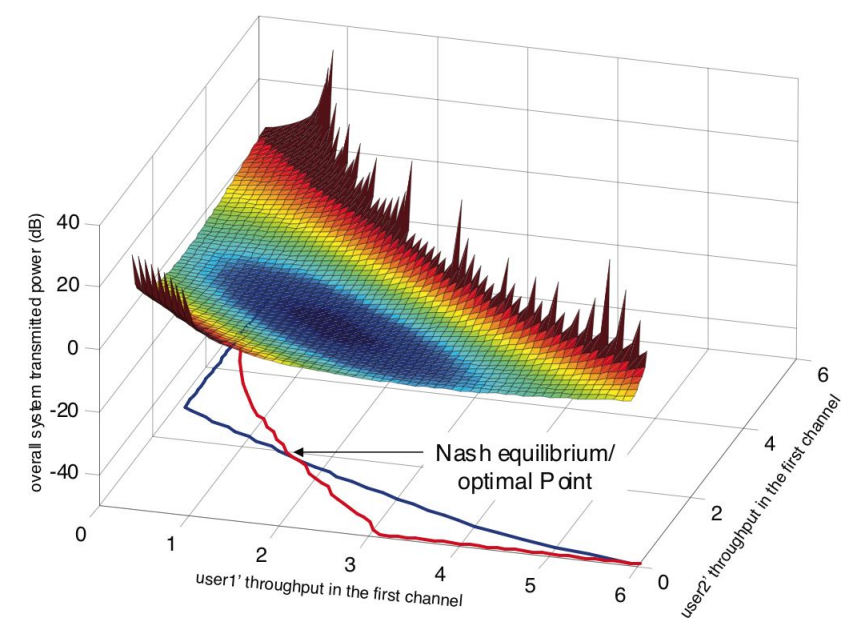

Fig. 1. Two-user Example: Unique NEP

\section{A. Two-User Two-sub-channel Example}

In this subsection, from the analysis of a two-user and twosub-channel example, we study the behaviors of the Nash equilibrium for (8) and optimal solution for (6). Then we provide the motivation why a virtual referee can improve the outcome of the game.

In the two-user and two-sub-channel example, two users have the desired rates of $R_{1}$ and $R_{2}$, respectively. Both users have the bandwidth of $W$. Suppose the $i^{t h}$ user puts $\alpha_{i}$ proportion of its rate to the first sub-channel. Define $\Lambda_{1}=$ $2^{\frac{\alpha_{1} R_{1}}{W}}$ and $\Lambda_{2}=2^{\frac{\alpha_{2} R_{2}}{W}}$. Define $\gamma_{i}^{l}$ as the minimal SINR requirement for the $i^{\text {th }}$ user's $l^{\text {th }}$ sub-channel. From (4), we have

$$
\begin{array}{r}
\gamma_{1}^{1}=\frac{\Lambda_{1}-1}{c_{3}}, \gamma_{1}^{2}=\frac{2 \frac{R_{1}}{w} / \Lambda_{1}-1}{c_{3}}, \gamma_{2}^{1}=\frac{\Lambda_{2}-1}{c_{3}}, \\
\text { and } \gamma_{2}^{2}=\frac{2 \frac{R_{2}}{w} / \Lambda_{2}-1}{c_{3}} .
\end{array}
$$

From (2), the corresponding transmitted power must satisfy

$$
P_{i}^{l} \geq \frac{\gamma_{i}^{l}\left(\sum_{k \neq i} P_{k}^{l} G_{k i}^{l}+N_{0}\right)}{G_{i i}^{l}}
$$

Notice that the optimum can be achieved when the equality holds [27] and $P_{i}^{l}$ is a function of $\alpha_{1}$ and $\alpha_{2}$. By using (2), (4) and (13), we know that the minimal SINR requirement must satisfy the following equations to make the system feasible.

$$
\gamma_{1}^{1} \gamma_{2}^{1}<\frac{G_{11}^{1} G_{22}^{1}}{G_{21}^{1} G_{12}^{1}} \text { and } \gamma_{1}^{2} \gamma_{2}^{2}<\frac{G_{11}^{2} G_{22}^{2}}{G_{21}^{2} G_{12}^{2}} .
$$

From system optimization point of view, for the example, the problem in (6) becomes

$$
\begin{aligned}
& \min _{0 \leq \alpha_{1}, \alpha_{2} \leq 1} P_{1}^{1}+P_{1}^{2}+P_{2}^{1}+P_{2}^{2} \\
& \text { s.t. } \sum_{j=1}^{2} P_{i}^{j} \leq P_{\max }, i=1,2 .
\end{aligned}
$$

From individual optimization point of view, the $i^{\text {th }}$ user tries to optimize the following constrained problem:

$$
\min _{0 \leq \alpha_{i} \leq 1} P_{i}^{1}+P_{i}^{2}
$$




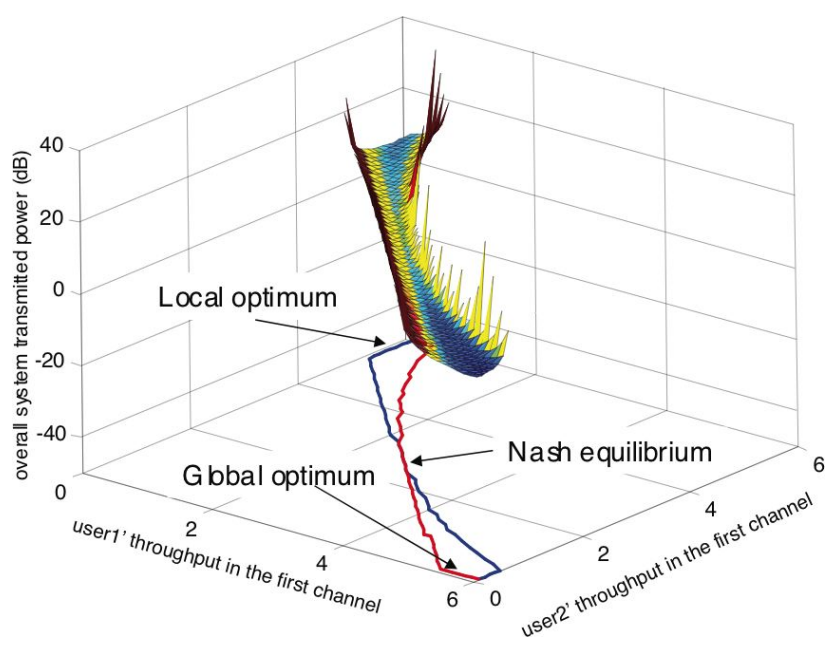

Fig. 2. Two-user Example: Multiple Local Optima

$$
\text { s.t. } P_{i}^{1}+P_{i}^{2} \leq P_{\max } \text {. }
$$

Nash equilibrium occurs when both users optimize (18). The remaining question is that if (17) and (18) produce the same results. The answer is not always by the following numerical example. The numerical example setup is: $\mathrm{BER}=10^{-3}, N_{0}=$ $10^{-3}, P_{\max }=10^{4}$,

$G^{1}=\left[\begin{array}{ll}0.0631 & 0.0100 \\ 0.0026 & 0.2120\end{array}\right]$, and $G^{2}=\left[\begin{array}{cc}0.4984 & 0.0067 \\ 0.0029 & 0.9580\end{array}\right]$

where $\left[\mathbf{G}^{l}\right]_{k i}=G_{k i}^{l}$. We have the following observations depending on the rate requirement.

- Fig. 1 shows the overall system power contour as a function of two users' rate allocations, where $R_{1}=R_{2}=$ 6 . The $\mathrm{x}$-axis and $\mathrm{y}$-axis are users' rates in the first subchannel, i.e. $\alpha_{i} R_{i}, i=1,2$, respectively. The $\mathrm{z}$-axis is the overall system transmitted power in $\mathrm{dB}$. The two curves show the locations for minimizing the two users' own powers when the interference from the other user is fixed, respectively. Each user tries to minimize its power by adjusting its rate allocation so that the operating point is more close to the curve. Consequently, the crossing point is a Nash equilibrium, where no user can reduce its power alone. We can see that the Nash equilibrium under this setup is unique and optimal for the overall system power. It is worthy to mention that the feasible region is not convex.

- Fig. 2 shows the situation when $R_{1}=R_{2}=8$. Because the rate is increased, each user has to increase his/her own power. Consequently, the co-channel interferences are increased since one user's power is the other's interferences. As a result, from the figure, the NEP is no longer a minimum for system overall power. There exist more than one local optima and the global optimum occurs when user 1 doesn't transmit on the sub-channel 1. If some mechanism can be implemented to prevent user 1 from using sub-channel 1, the game can converge to the global optimum for system optimization.

- Fig. 3 shows the situation when $R_{1}=R_{2}=8.5$. The contour graph is no longer connected. There are two NEPs and two local optima for system overall power.

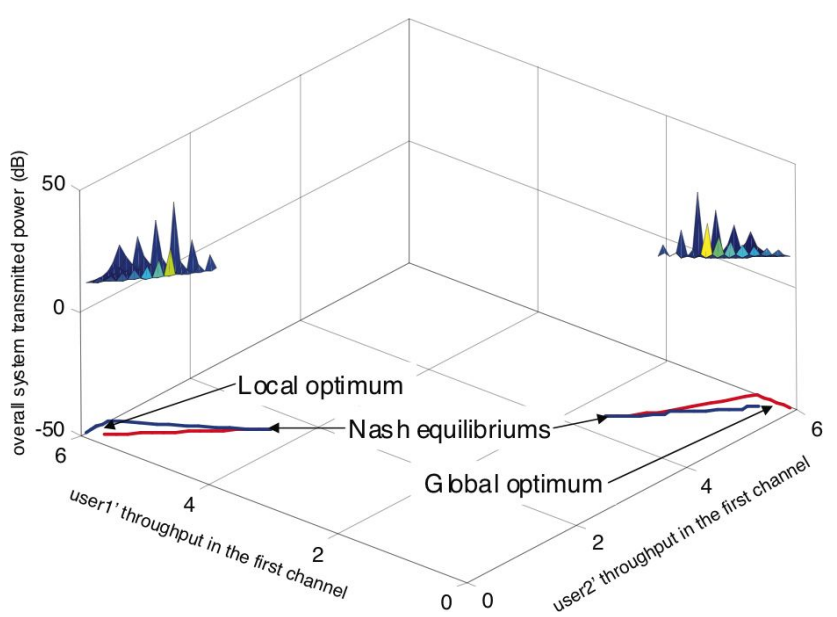

Fig. 3. Two-user Example: Multiple NEPs

- If we further increase $R_{1}=R_{2}=10$, there exists no feasible region, i.e., both users cannot have a resource allocation that satisfies both power and rate constraints. In this case, the rate requirement should be reduced so that the interferences also reduced.

From the above observations, we can conclude that the behaviors of the optimal solution in (6) and NEP in (8) depend on how high the required rates are, i.e. how severe the interferences are. In many cases, the game converges to some undesirable NEP with bad performances. In order to improve the performances of the NEP, we propose a referee to monitor and mediate the game. First, a criterion is found to decide whether the users can efficiently share the sub-channels, i.e. whether or not the NEP is desirable. If not, the referee would mandatorily modify the game rule by preventing some users from using the resources such as sub-channels or reducing the required transmission rate, so that the rest of users can share the resources more efficiently. Before we develop the proposed algorithm, three lemmas are proved for the NEP in the next subsection.

\section{B. Lemmas of Nash Equilibrium}

In this subsection, we prove the existence of NEP and conditions for NEP to be optimal by the following three Lemmas. Note that the proofs are shown in the Appendix.

Lemma 1: There exists an NEP in the proposed game defined in (8), if $\Omega$ is not empty.

In the following Lemma, we show a sufficient condition when system optimization equals individual optimization.

Lemma 2: The optimal system optimization point is an NEP for two-user two-sub-channel case, when the rate requirement is satisfied, the overall power is less than $P_{\text {sum }}$, and both users transmit on both sub-channels.

Next, we will show a necessary condition when system optimization equals individual optimization under the system model proposed in Section II.

Lemma 3: If the global minimum of (6) occurs when $r_{i}^{l}>$ $0, \forall A_{i l} \neq 0$ and $\sum_{l=1}^{L} P_{i}^{l}<P_{\max }$ and $\sum_{l=1}^{L} r_{i}^{l}=R_{i}, \forall i$, the NEP satisfies the necessary Karush-Kuhn-Tucker (KKT) condition [26]. 


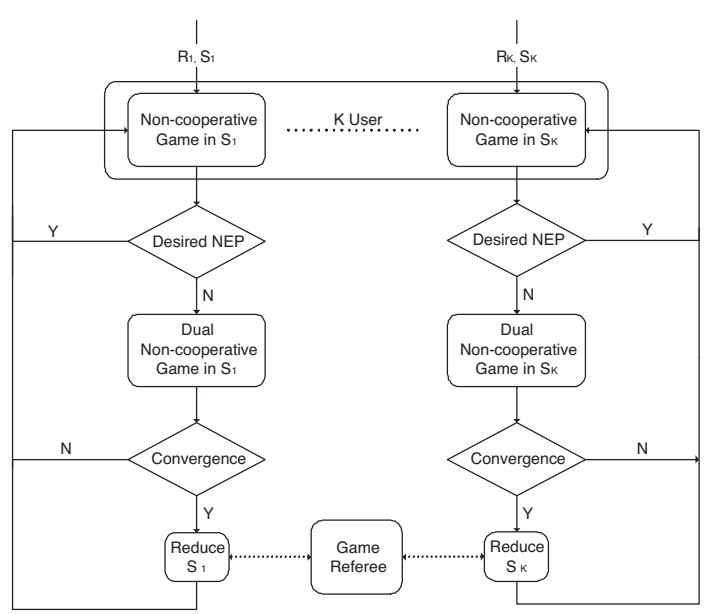

Fig. 4. Non-cooperative Game with a Referee

Notice that the above Lemma is a necessary condition, i.e., we cannot say that system optimization equals to individual optimization under the above conditions. However, we are sure that if system optimization does not equal to individual optimization, the above conditions do not hold. So by using this fact, we propose the condition for a virtual referee to mediate the game in the next subsection.

\section{Distributed Resource Allocation Algorithm with a Virtual Referee}

Before developing the proposed algorithm, we analyze two special resource allocation schemes. In the first scheme, the groups of sub-channels are assigned to different cells without overlapping such that there are no co-channel interferences among the different cells. In this case, $\sum_{l}[\mathbf{A}]_{i l}=1, \forall i$. We call it the fixed channel assignment scheme. However, this method has the disadvantages of low spectrum efficiency because of the low frequency re-usage. The overall transmitted power in (6) solved by this method is far from minimum, because it doesn't take the advantage of the multiuser diversity and power control. In the second scheme, all the users share all the sub-channels, i.e., $[\mathbf{A}]_{i l}=1, \forall i, l$. We call it iterative waterfilling scheme, which is basically the pure non-cooperative game. From Fig. 2 and Fig. 3, we can see that the system can be balanced at the undesirable point, because of the severe inter-cell co-channel interferences. Such facts motivate us to believe that the optimal resource allocation is between these two special schemes, i.e., each sub-channel can be shared by only a group of users for transmissions $\left(1 \leq \sum_{l}[\mathbf{A}]_{i l} \leq\right.$ $L, \forall i)$. To decide who should share these sub-channels and who should not, we introduce a virtual referee to the noncooperative game.

The basic idea of the proposed scheme is to introduce a virtual referee to heuristically improve the outcome of the non-cooperative game. Each distributed user plays the noncooperative game to optimize his/her own resource usage among the different sub-channels. If the outcome of the game is the desired NEP and satisfies all user's power and rate constraints, the referee will do nothing. Otherwise, the referee will restrict some users from using some sub-channels. By doing this, the game rule will be changed so as to improve the outcome of the game.

Specifically, in Fig. 4, we show the block diagram of the proposed algorithm. We define the sub-channel set that the $i^{\text {th }}$ user can allocate their rates as transmission group $S_{i}$. We initially set $S_{i}$ to have all the sub-channels, i.e. $\{1, \ldots, L\}$. Then the non-cooperative game in (8) is applied. If all users' required rates are achieved and each user's overall power is less than the maximal power as the Lemma 3 requires in the previous section, the outcome of the game is considered to be the desired NEP. Under this condition, each user continues the non-cooperative game to adapt to the channel fluctuations.

If users' request rates are too high, the non-cooperative game might not be able to converge due to the fact that there might be no feasible solution discussed in Section IV.A. Under this condition, no matter how the users adapt their resource allocation strategies, there will be no operation points where all users can transmit simultaneously while achieving their rate requirements. The system will oscillate as a result of infeasibility. To improve the feasibility, the users, whose power achieves the maximal power while the required rates are not satisfied, will play the dual non-cooperative game defined as follows:

\section{Dual Non-Cooperative Game:}

$$
\max _{\mathbf{r}_{i} \in \Omega} \sum_{l \in S_{i}} r_{i}^{l}, \text { s.t. } \sum_{l \in S_{i}} P_{i}^{l}=P_{\max } .
$$

The rationale is to achieve the largest transmission rate with the limited power resources. The user automatically switches to the dual game whenever its power reaches the maximal power.

The users select to play the two types of games in (8) or (19) depending whether or not the requested rate can be achieved by the maximal power. The following Lemma proves the convergence of the two games playing simultaneously by different users.

Lemma 4: There exists the NEP for multiple users playing the different games defined in (8) and (19).

After the games converge, if any user has to play the dual non-cooperative game, we assume the NEP is not desired, so that some user must remove some sub-channels from his/her transmission group. If the removal can make all users balanced at the desirable NEP, the algorithm continues in the noncooperative game step, i.e. all users play the game in (8). Otherwise, we continue the user removal step, until no user can be removed, performances cannot be improved, or the desirable NEP is achieved.

The criterion for the user to remove a specific sub-channel is determined by the channel gain and the interferences plus noise level. If user $i$ cannot satisfy his/her constraints, the users who share the sub-channels in $S_{i}$ will decide who will be prevented from using which sub-channel. The sub-channel with smallest channel gain and largest interferences plus noise will be selected, i.e., the $j^{\text {th }}$ user will drop the $l^{\text {th }}$ sub-channel if

$$
(l, j)=\arg \min _{l, j} \frac{P_{j}^{l} G_{j j}^{l}}{\sum_{k \neq j} P_{k}^{l} G_{k j}^{l}+N_{0}}
$$

where $l \in S_{i}$ and user $j$ shares a least one sub-channel with user $i$. 
TABLE I

Distributed Resource Allocation Algorithm

\begin{tabular}{|c|}
\hline $\begin{array}{l}\text { 1. Initialization: } \\
\quad R_{i}=\text { predefined value, } S_{i} \text { includes all sub-channels. }\end{array}$ \\
\hline $\begin{array}{l}\text { 2. Non-cooperative Game: } \\
\text { each user have the non-cooperative game in (8). }\end{array}$ \\
\hline $\begin{array}{l}\text { 3. Desirable NEP? } \\
\text { if } \sum_{l=1}^{L} P_{i}^{l}<P_{\max } \text { and } \sum_{l=1}^{L} r_{i}^{l}=R_{i} \\
\text { occupy every sub-channel, go to step } 2 \\
\text { otherwise, go to step } 4 \text {. }\end{array}$ \\
\hline $\begin{array}{l}\text { 4. Dual Non-cooperative Game } \\
\text { for users with } \sum_{l=1}^{L} P_{i}^{l}=P_{\max } \text { and } \sum_{l=1}^{L} r_{i}^{l}<R_{i} \text {, } \\
\text { play (19) instead. } \\
\text { if game converges, go to step 5, otherwise step } 2 \text {. }\end{array}$ \\
\hline $\begin{array}{l}\text { 5. Sub-channel Removal/Rate Reduction: } \\
\text { remove sub-channel from transmission group } \\
\text { by }(20) \text {, then go to step } 2 \text {. } \\
\text { if no user can reduce transmission group or no } \\
\text { performance improvement, go to step } 2 \text {. }\end{array}$ \\
\hline
\end{tabular}

The criterion for whether or not the user can be removed from the transmission group is determined by three factors.

1) Each user must have at least one sub-channel to transmit, i.e., $[\mathbf{A}]_{i l}=1, \exists l, \forall i$.

2) No sub-channel is wasted, i.e., at least one user is assigned for each sub-channel. $[\mathbf{A}]_{i l}=1, \exists i, \forall l$.

3) User cannot be prevented from using the sub-channel, if the user cannot transmit its rate $R_{i}$ using the rest of subchannels within maximal power limitation, even though he/she occupies them alone.

The referee is a virtual concept and can be implemented in any base station among the co-channel cells to coordinate the resource usage. In order to apply the proposed algorithm, we assume that base stations can accurately measure the channel gains and interferences plus noise power. Moreover there are reliable feedback channels to mobiles for the referee's decisions. All these assumptions are similar to close-loop power control and are reasonable for implementation in practice. The proposed distributed algorithm for each cell is shown in Table I.

\section{Complexity, Convergence, and Implementation}

In this subsection, we discuss some practical issues for the proposed scheme with a virtual referee. The complexity of the proposed non-cooperative game scheme is $O(L \log L)$, which is the complexity of water-filling in (10) [2]. The convergence speed of the non-cooperative game is determined by the largest eigenvalue of $\mathbf{D}^{l} \mathbf{F}^{l}$, from Perron-Frobenius theorem [30]. This can also be illustrated by the example in Fig. 1 . The convergence speed depends on the two curves where the best responses of one user are occurred conditioned on the other users' rates. Starting from any feasible point, in each round, two users try to move the operating point vertically and horizontally to the corresponding curves. Consequently, the solution is getting closer to the optimal after each round. If the two curves are too parallel to each other, the convergence speed will be slow. It is worth mentioning that the convergence of the non-cooperative game is similar to the convergence of close-loop power control proposed in [28] and [29]. Some fast convergence algorithms can also be employed such as second order power control proposed in [31]. The frequency of applying the proposed scheme depends not only on the convergence speed, but also on how fast channels change. From the current 3G system, this frequency is about 1500 times per second. For the multiple cell wireless LAN application where users moves less frequently, the frequency can be much lower. Since the complexity of the algorithm is very low, the computation burden is not high for the network.

For centralized schemes, the channel information of all users are required at all times in order to perform the optimization. For the proposed scheme, the overhead and signaling occur only when the system cannot be balanced at a good Nash Equilibrium. Under this condition, a referee needs to collect information from all the co-channel interfered cells. The frequency for this overhead and signaling is much lower than that required for centralized schemes. The collected information includes power value $P_{j}^{l}$, channel gain value $G_{j j}^{l}$, and noise-plus-interference variance value $\sum_{k \neq j} P_{k}^{l} G_{k j}^{l}+N_{0}$ over all sub-channels. Since all these values are consistently measured by all the distributed users at any time, there is no need for extra measurement. The amount of these information is also small and can be exchanged among the cells with few packets. So the overhead and signaling are negligible.

There are some other implementation issues such as synchronization. For the downlink case, the synchronization can be achieved by utilizing Global Position System in the BS. For the uplink case, the carrier frequencies can have up to $10 \mathrm{~Hz}$ differences, which will cause the inter-sub-channelinterferences. Consequently, there might be some error floor in the BER performances of MQAM. This problem can be alleviated by feeding back the frequency offset. Moreover, in [32], guard sub-channel is put at the edge of each sub-channel such that multiple access interference can be minimized and synchronized algorithm is applicable for each sub-channel.

It is also worth mentioning that if the distributed users can take into account of the referee's rule as well, they can gain advantages and the game equilibrium will be changed. However, we do not focus on how to combat the greedy player in this paper. Instead, the proposed scheme targets on the distributed resource allocation so that the overhead and signaling can be reduced. In other words, for example, the distributed users might belong to the same company and it is not necessary to design clever schemes to take advantages of the referee scheme of the same company.

\section{Simulation Results}

To evaluate the performances of the proposed scheme, we set up the simulations consisting of a two-cell case and a seven-cell case. We compare the proposed referee-based scheme with two other schemes. The first scheme is the fixed channel assignment algorithm which always has only one user per sub-channel. The second scheme is iterative water-filling algorithm which is basically the non-cooperative game without a referee. In the following, the simulation results for a twocell system are presented in the first subsection, and those for multi-cell system are shown in the second subsection. 


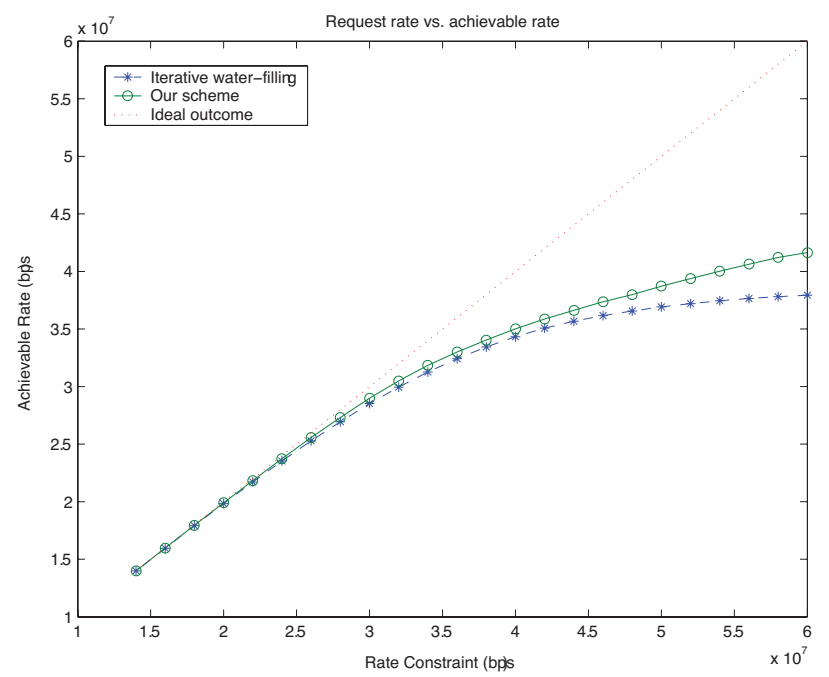

Fig. 5. Achievable Rate versus Desired Rate (per User) for the Two-Cell Case

\section{A. Two Cell Case}

In the two-cell case, one base station is located at the center of each cell and one mobile per cell is generated as a uniform distribution within the corresponding cell. The propagation model assumes the operation in a suburban environment and takes into consideration of path loss and shadowing. The received signal (in $\mathrm{dB}$ ) at distance $d$ from the base station is $L(d)=L\left(d_{0}\right)+10 \alpha \log _{10} \frac{d}{d_{0}}$, where $d_{0}=10 \mathrm{~m}$ is used as a reference point in measurements $\left(L\left(d_{0}\right)=0 \mathrm{~dB}\right)$ and $\alpha$ is set to 3.5. Shadow fading for each user is modelled as an independent log-normal random variable with standard deviation $\sigma=10 \mathrm{~dB}$. The four-path Rayleigh model is taken into consideration to simulate the frequency selective fading channels, which has an exponential power profile with 100ns rootmean-square (RMS) delay spread. We consider an OFDMA system with 32 sub-channels in total. The overall bandwidth is $6.4 \mathrm{MHz}$. The total transmission power for every mobile is constrained by a maximal value of $5 \mathrm{~mW}$. The receiver thermal noise is $-70 \mathrm{dBmW}$. The BER of the transmitted symbols is required to be $10^{-3}$ on every sub-channel for each user, which corresponds to $c_{3}=0.2831$ in (4). Two cells are located adjacent to each other.

In Fig. 5, we show the achievable rate versus the required rate for the proposed scheme and the pure water-filling scheme. Here we assume $R_{1}=R_{2}$. For comparison reason, we also show the ideal case where the achievable rate equals to the required rate. When the required rate is small, both users can achieve the desired rate while minimizing their own power. Under this condition, the dual game is seldom played. Consequently, the achievable rate is the same as the required rate. When the required rate is high, the system might not be feasible under all conditions. As a result, some users turn to play the dual game and the achievable rate is smaller than the desired rate. Compared to the iterative water-filling scheme, the proposed scheme has up to $10 \%$ higher achievable rates. This is due to the fact that the resource usage of crowed subchannels has been improved by the referee.

In Fig. 6, we show the overall transmitted power versus achievable rate $R_{i}$. When the rate requirement is increas-

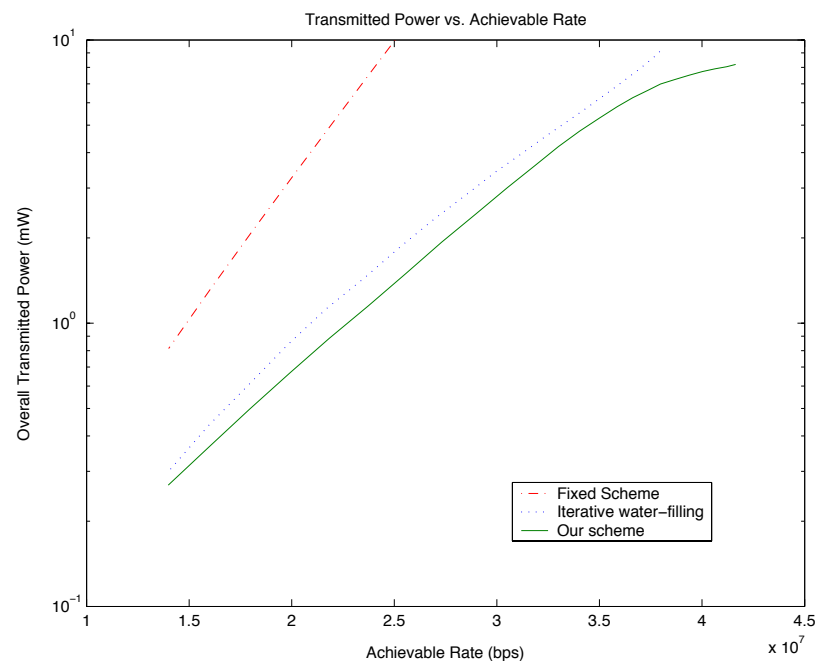

Fig. 6. Total Power versus Achievable Rate for the Two-Cell Case

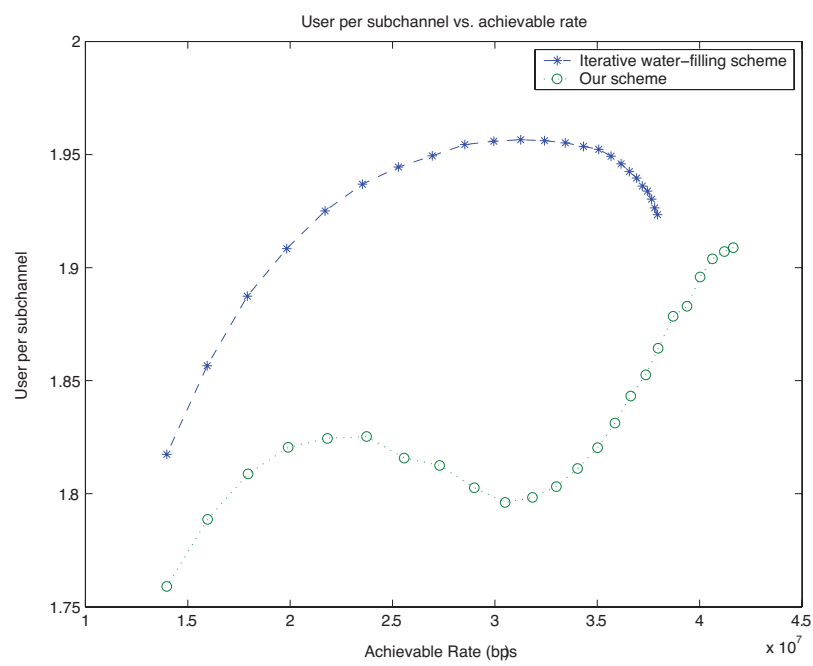

Fig. 7. User Per Sub-channel versus Achievable Rate for the Two-Cell Case

ing, the transmit power is increasing and the co-channel interferences become more severe. Notice that the power axis is plotted in log scale. Since the curves are linearly increasing when the rate is small, the transmit power increases exponentially with the rate constraint. The reason is that the rate is a $\log$ function of power in (4). Compared with the fixed assignment algorithm, the proposed algorithm reduces up to $80 \%$ of powers. This is because the fixed assignment algorithm wastes many resources by letting only one user occupy any sub-channel. Compared with the pure iterative water-filling algorithm, the proposed algorithm reduces about $25 \%$ of powers. The reason is that the proposed refereebased scheme can improve the Nash equilibrium of the noncooperative game. Notice that the maximal achievable rate for the proposed scheme is higher than both fixed scheme and iterative water-filling scheme. For the large values of achievable rate, the overall transmission power does not follow the linear increase and saturates at $P_{\max }$, because most of the users play the dual game when the rate is high. 


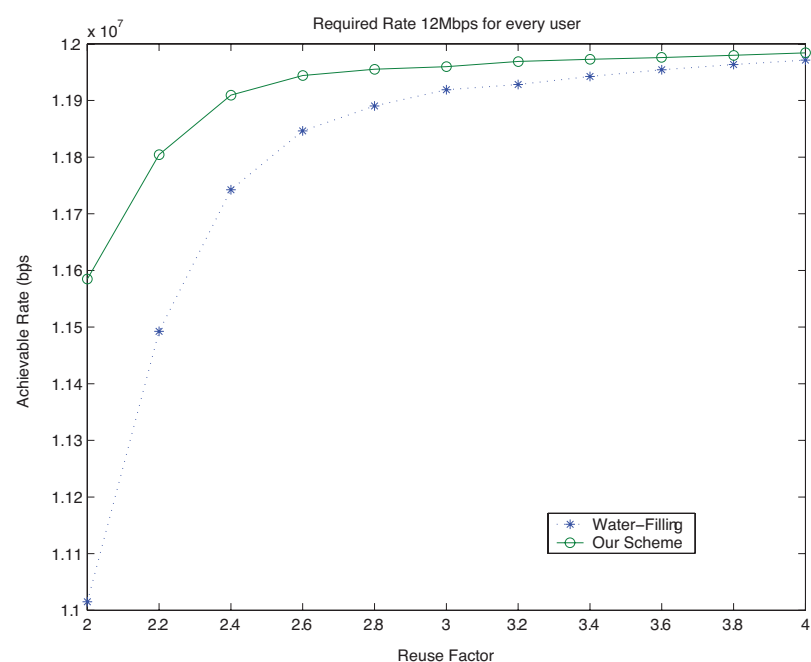

Fig. 8. Achievable Rate versus $R_{u}$ for the Multicell Case

In Fig. 7, we show the number of users per sub-channel versus rate constraint. ${ }^{2}$ The iterative water-filling has average number of user per sub-channel from 1.86 to 1.96 , the proposed scheme from 1.76 to 1.92 , and the iterative waterfilling always has higher number of user per sub-channel than the proposed scheme. The user per sub-channel of the iterative water-filling algorithm is increasing when the rate requirement is less than $30 \mathrm{Mbps}$. This is because some subchannels may not have been allocated powers when the rate constraint is small. As the rate constraint increases, the users are greedier to compete for resources when the rates and cochannel interferences are high. When the rate is higher than $30 \mathrm{Mbps}$, the user per sub-channel for iterative water-filling scheme starts to decrease. Because the dual game is played under this condition, the user does not allocate the limited power to the sub-channels with high interferences.

The proposed algorithm has larger user per sub-channel when the rate requirement is small. This is because of the same reason as the pure iterative water-filling. But when the rate becomes larger, the user per sub-channel is reduced. The reason is that more users are removed by the referee from using the sub-channel when the rate constraint is large. Consequently, the users can utilize the limited resources more efficiently. The gap between the proposed scheme and the pure water-filling scheme is larger when the rate constraint is large and the rate is less than 30Mbps. This is because some sub-channels can only support one user especially when the rate constraint and co-channel interferes are large, while the iterative water-filling algorithm still tries to put two users into one sub-channel. When the rate is larger than $30 \mathrm{Mbps}$, the number of user per sub-channel for the proposed scheme is increasing, because most of users turn to play the dual game. Under this condition, the power, rate, and sub-channel allocation almost achieves the boundary of the feasible range for the system to support users' rates. The proposed referee based approach has less room to improve the system performances.

\footnotetext{
${ }^{2}$ The fixed channel assignment algorithm always has one user per subchannel. So it is not shown in the figure.
}

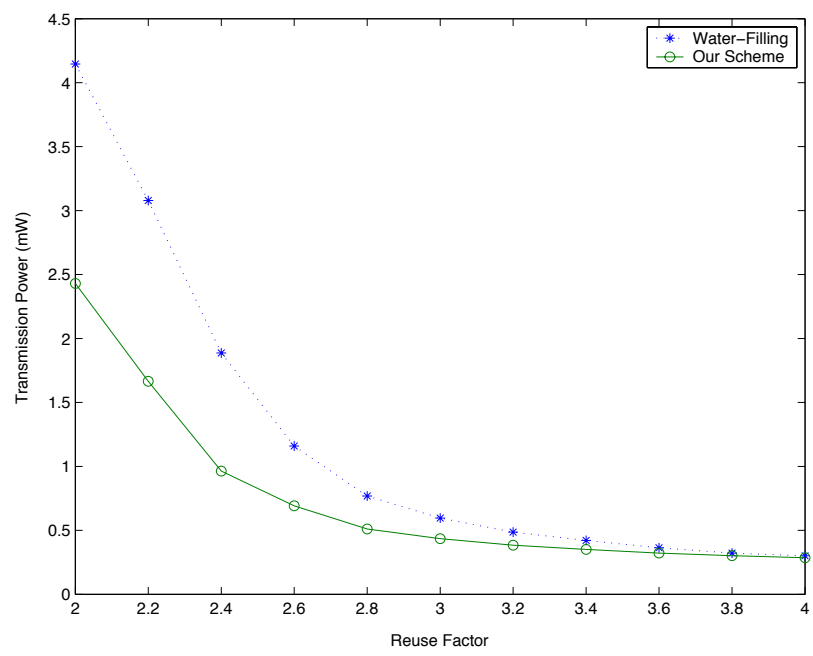

Fig. 9. Average Power versus $R_{u}$ for the Multicell Case

\section{B. Multi-Cell Case}

Multi-cell case simulation with seven users is conducted. One cell is located in the middle and the other six cells are located at the angle of $[0,30,90,150,210,270]$ degrees, respectively. The cell radius is $r=100 \mathrm{~m}$. The rate constraint is $12 \mathrm{Mbps}$ for each user and the power constraint is $P_{\max }=$ $10 \mathrm{~mW}$. We define the reuse factor $R_{u}$ as the distance between two base stations $D$ over the cell radius $r$. The smaller reuse distance, the more severe the co-channel interferences are. The other settings are the same as those of two-cell case.

In Fig. 8, Fig. 9, and Fig. 10, we show the achievable rate, average transmitted power, and users per sub-channel versus reuse distance for the iterative water-filling algorithm and the proposed refereed-based algorithm, respectively. The cochannel interferences are more severe when the reuse distance is small. The proposed scheme can achieve higher feasible rate compared to iterative water-filling scheme. The gap between required rate and achievable rate is larger when $R_{u}$ becomes smaller. This is because more users have to play dual noncooperative game when the co-channel interferences are large. Notice that the rate gap is relatively small compared to the requested rate. In Fig. 9, we can see that the proposed algorithm can reduce the transmit power by about $40 \%$ when the co-channel interferences are severe (e.g. $R_{u}=2$ ), which will greatly improve the system performance. The power reduction is due to the reason that the proposed scheme removes more users and reduces number of users per sub-channel as shown in Fig. 10. Consequently, the usage of the resources can be more efficient. On the other hand, when $R_{u}$ is increasing, the co-channel interferences are reduced and the channel is impaired more by noises than by interferences. As the result, two schemes shows the similar achievable rate, transmitted power, and user per sub-channel. Since the effects of others' strategies are smaller under this situation, the virtual referee seldom works. Therefore the proposed scheme reduces to the iterative water-filling scheme.

\section{CONCLUSions}

In this paper, we develop a distributed game theory approach with a referee to adaptively assign the sub-channels, 


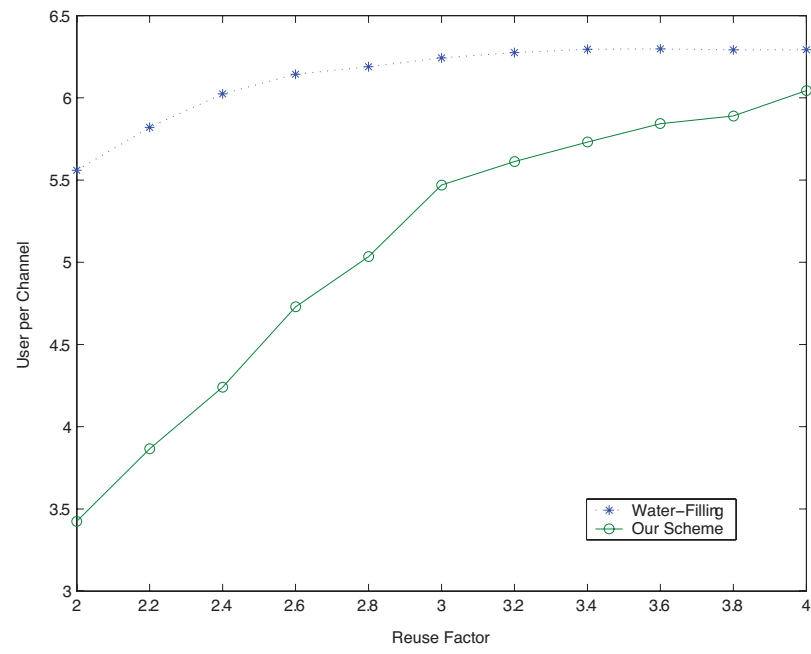

Fig. 10. User Per Sub-channel versus $R_{u}$ for the Multicell Case

rates, and power for multi-cell OFDMA networks. The goal is to minimize the total transmission power under the constraints of the desirable rate and the maximal transmitted power. To improve the performances, a virtual referee is introduced to the networks to regulate the competition for the resource usage. Compared with the iterative water-filling method, the proposed scheme has one more optimization dimension for channel assignment of sub-channel sharing. Moreover, this refereebased scheme imposes little burden on system implementation. From the simulation results, the proposed distributed algorithm reduces the transmitted power by up to $80 \%$ and $25 \%$ compared with the fixed assignment scheme and the iterative water-filling scheme for two-cell case, respectively. The achievable rate can be improved up to $10 \%$. As for the multi-cell case, the proposed scheme saves up to $40 \%$ power compared with the iterative water-filling scheme when the cochannel interferences are severe.

\section{APPENDIX}

\section{A. Proof of Lemma 1}

In [8], it has been shown an NEP exists, if for all $i$, the following two conditions can be satisfied

1) $\Omega$, the support domain of $u_{i}\left(\mathbf{r}_{i}\right)$, is a nonempty, convex, and compact subset of some Euclidean space $\Re^{L}$.

2) $u_{i}\left(\mathbf{r}_{i}\right)$ is continuous in $\mathbf{r}_{i}$ and quasiconvex in $\mathbf{r}_{i}$.

Since each sub-channel can be allocated by $P_{\max }$ and overall transmitted power for all sub-channels is linearly constrained by $P_{\max }$, the supporting domain for power allocation is compact and convex. Because the rate is a concave function of transmitted power if the interferences are fixed, the supporting domain $\Omega$ for $r_{i}^{l}, \forall l$ is a convex and compact subset of some Euclidean space $\left(\Re^{+}\right)^{L}$. It is worthy mentioning that $\Omega^{K}$ is not convex and one example is shown in Fig. 1. But our proof only needs that $\Omega$ is convex and nonempty.

From (2) and (4), when the water-filling is done for (8),

$$
u_{i}=\sum_{l=1}^{L}\left(\frac{\left(2^{r_{i}^{l}}-1\right)\left(\sum_{k \neq i} P_{k}^{l} G_{k i}^{l}+N_{0}\right)}{c_{3}^{i} G_{i i}^{l}}-\mu_{i} r_{i}^{l}\right) \text {. }
$$

Obviously, $u_{i}$ is continuous for $\mathbf{r}_{i}$ and convex for $r_{i}^{l}$. To prove the jointly quasi-convex in $\mathbf{r}_{i}$, we need to define $u_{i}=+\infty$ if infeasible. Suppose there are two operation points $\mathbf{r}_{i}$ and $\mathbf{r}_{i}^{\prime}$. If the linear combination between the two operation points is located within feasible range, from (21), the convexity can be easily proved. Otherwise, $u_{i}=+\infty$ which is larger than any linear combination between $u_{i}\left(\mathbf{r}_{i}\right)$ and $u_{i}\left(\mathbf{r}_{i}^{\prime}\right)$. So both conditions hold.

\section{B. Proof of Lemma 2}

When the rate requirement is satisfied and the overall power is less than $P_{\text {sum }}$, the constraints in (17) and (18) can be omitted. The boundary conditions for $\alpha_{1}$ and $\alpha_{2}$ can also be omitted. The Lagrangian multiplier for minimizing system overall power in (17) can be written as:

$$
J\left(\alpha_{1}, \alpha_{2}\right)=P_{1}^{1}+P_{1}^{2}+P_{2}^{1}+P_{2}^{2}
$$

The Lagrangian multiplier for individual to minimize the power in (18) can be written as:

$$
J_{1}\left(\alpha_{1}\right)=P_{1}^{1}+P_{1}^{2} \text { and } J_{2}\left(\alpha_{2}\right)=P_{2}^{1}+P_{2}^{2} .
$$

By substituting $\frac{\partial J_{1}}{\partial \alpha_{1}}=0$ and $\frac{\partial J_{2}}{\partial \alpha_{2}}=0$ into $\frac{\partial J}{\partial \alpha_{1}}=0$ and $\frac{\partial J}{\partial \alpha_{2}}=0$, we have

$$
\left\{\begin{array}{l}
\frac{\gamma_{1}^{1}}{\gamma_{1}^{2}}=\frac{G_{11}^{1} G_{21}^{2}}{G_{11}^{2} G_{21}^{1}} \\
\frac{\gamma_{2}^{1}}{\gamma_{2}^{2}}=\frac{G_{22}^{1} G_{12}^{2}}{G_{22}^{2} G_{12}^{1}}
\end{array}\right.
$$

The above equalities in (24) can also be derived from $\frac{\partial J_{1}}{\partial \alpha_{1}}=0$ and $\frac{\partial J_{2}}{\partial \alpha_{2}}=0$. So we prove that the NEP where $\frac{\partial J_{1}}{\partial \alpha_{1}}=0$ and $\frac{\partial J_{2}}{\partial \alpha_{2}}=0$ is optimal for (6) where $\frac{\partial J}{\partial \alpha_{1}}=0$ and $\frac{\partial J}{\partial \alpha_{2}}=0$.

\section{Proof of Lemma 3}

First, if at NEP, no user can improve his/her own performance by changing his/her rates alone. If $\sum_{l=1}^{L} P_{i}^{l}<$ $P_{\text {max }}$ and $\sum_{l=1}^{L} r_{i}^{l}=R_{i}, \forall i$ at NEP and if the interferences are considered as noises, the resource allocation is optimal for each user. By Lagrangian method, define $\nabla=\frac{\partial}{\partial \mathbf{r}_{i}}$, the following equation hold at the NEP when power is less than $P_{\max }$.

$$
\nabla\left(\sum_{l=1}^{L} P_{i}^{l}\right)-\mu_{i} \nabla\left(\sum_{l=1}^{L} r_{i}^{l}-R_{i}\right)=0 .
$$

The above equation is for each user's optimization in (8). For the system optimization problem in (6), if $r_{i}^{l}>0, \forall A_{i l} \neq 0$ and $\sum_{l=1}^{L} P_{i}^{l}<P_{\max }, \forall i$, the global optima will satisfy the KKT condition without considering the inequality constraints:

$$
\sum_{i=1}^{K} \nabla\left(\sum_{l=1}^{L} P_{i}^{l}\right)-\sum_{i=1}^{K} \mu_{i} \nabla\left(\sum_{l=1}^{L} r_{i}^{l}-R_{i}\right)=0 .
$$

Obviously, when the non-cooperative game in (8) converges to NEP, (26) will be satisfied from (25). So the KKT necessary condition is satisfied at the NEP. 


\section{Proof of Lemma 4}

To prove the convergence, we need to first show that the feasible range is not empty and then there exits an NEP if both games are played together. From the analysis in Section II-D, we know that the feasible range is not empty as long as the required rates are small enough. In the proposed two-game scenario, if any user detects that it is impossible to achieve the desired rate by the maximal power, the user will change the resource optimization to maximize the achievable rate by the limited power. Consequently, the rate is reduced. In the worst case, all users play the dual non-cooperative game. Under this condition, a feasible solution can be surely achieved since all users' power is bounded.

Starting from this feasible solution, we need to prove both games satisfy the two conditions in Lemma 1. Obviously the support domain for the dual game in (19) is nonempty, convex, and compact subsect of some Euclidean Space $\Re^{L}$. The game utility $u_{i}$ in (19) is linear function of $r_{i}^{l}$ and consequently quasi-convex in $\mathbf{r}_{i}$. Consequently, there exists an NEP if both games are played together.

\section{REFERENCES}

[1] W. Yu, G. Ginis, and J. M. Cioffi, "Distributed multiuser power control for digital subscriber lines", IEEE Journal on Selected Areas in Commun., vol.20, no.5, pp.1105-1114, June 2002.

[2] W. Yu, W. Rhee, S. Boyd, and J.M. Cioffi, "Iterative water-filling for gaussian vector multiple access channels", IEEE Transactions on Information Theory, vol.50, no.1, pp.145-151, January 2004.

[3] P. Viswanath, D. Tse, and V. Anantharam, "Asymptotically optimal waterfilling in vector multiple access channels", IEEE Transactions on Information Theory, vol.12, no.4, pp.241-267, Janunary 2001.

[4] O. Kaya and S. Ulukus, "Optimum power control for fading CDMA with deterministic sequences", in 40th Annual Allerton Conference on Communications, Control and Computing, Allerton, IL, October 2002.

[5] H. J. Su and E. Geraniotis, "A distributed power allocation algorithm with adaptive modulation for multi-cell OFDM sytems," in 5th IEEE International Symposium on Spread Spectrum Techniques and Applications, vol.2, pp.474-478, Sun City, South Africa, September, 1998.

[6] G. Li and H. Liu, "Downlink dynamic resource allocation for multi-cell OFDMA system", in Conference Record of the Thirty-Seventh Asilomar Conference on Signals, Systems and Computers, 2003, vol.1, pp.9-12, Pacific Grove, CA, November 2003.

[7] I. Koutsopoulos and L. Tassiulas, "Channel state-adaptive techniques for throughput enhancement in wireless broadband networks," in Proceedings of Twentieth Annual Joint Conference of the IEEE Computer and Communications Societies (INFOCOM), pp.757-766, Anchorage, AK, April 2001.

[8] D. Fudenberg and J. Tirole, Game theory, MIT Press, Cambridge, MA, 1991.

[9] C. U. Saraydar, N. B. Mandayam, and D. J. Goodman, "Pricing and power control in a multicell wireless data network," IEEE Journal on Selected Areas on Commun., vol.19, no.10, pp.1883-1892, October 2001.

[10] T. Roughgarden, Selfish routing and the rrice of anarchy, MIT Press, 2005.

[11] P. Liu, P. Zhang, S. Jordan, and M. L. Honig, "Single-cell forward link power allocation using pricing in wireless networks", IEEE Transactions on Wireless Communications, vol.3, issue:2, pp.533-543, March 2004.

[12] L. Buttyan and J. P. Hubaux, "Stimulating cooperation in self-organizing mobile ad hoc networks," ACM Journal for Mobile Networks (Monet), special issue on Mobile Ad Hoc Networks, summer 2002.

[13] S. Zhong, R. Y. Yang, and J. Chen, "Sprite: a simple, cheat-proof, creditbased system for mobile ad-hoc networks," in Proceedings of The 22nd Annual Joint Conference of the IEEE Computer and Communications Societies ( INFOCOM'03), San Francisco, CA, March 2003.

[14] P. Michiardi and R. Molva, "A game theoretical approach to evaluate cooperation enforcement mechanisms in mobile ad-hoc networks," in Proceedings of Modeling and Optimization in Mobile, Ad Hoc and Wireless Networks (WiOpt'03), INRIA Sophia-Antipolis, France, March 2003.
[15] W. Wang, X. Li, and Z. Sun, "Design differentiated service multicast with selfish agents", IEEE Journal of Selected Area in Communications, vol.24, no.5, pp.1061-1073, May 2006

[16] H. Yaiche, R. R. Mazumdar, and C. Rosenberg, "A game theoretic framework for bandwidth allocation and pricing in broadband networks", IEEE/ACM Transactions on Networking, vol.8, no.5, pp.667-678, October 2000.

[17] Z. Han, Z. Ji, and K. J. R. Liu, "Fair multiuser channel allocation for OFDMA networks using Nash Bargaining and coalitions", IEEE Transactions on Communications, vol.53, no.8, pp.1366-1376, August 2005

[18] M. H. Halldorson, J. H. Halpern, L. Li, and V. S. Mirrokni, "On spectrum sharing games", in Proceedings of ACM Symposium on Principle of Distributed Computing (PODC), pp.107-114, St. John's, Newfoundland, Canada, July 2004.

[19] L. Cao, H. Zheng, "Spectrum allocation via local bargaining in ad hoc networks", in Proceedings of Second Annual IEEE Communications Society Conference on Sensor and Ad Hoc Communications and Networks (SECON), Santa Clara, CA, September 2005.

[20] M. Felegyhazi, J. P. Hubaux and L. Buttyan, "Nash Equilibria of Packet Forwarding Strategies in Wireless Ad Hoc Networks", IEEE Transactions on Mobile Computing, volume 5, number 5, May 2006.

[21] Z. Han, C. Pandana, and K. J. Ray Liu, "A self-learning repeated game framework for optimizing packet forwarding", IEEE Wireless Communications and Networking Conference, p.p.2131 - 2136, vol.4, New Orleans, 2005.

[22] Z. Han, Z. Ji, and K. J. R. Liu, "Power minimization for multi-cell OFDM networks using distributed non-cooperative game approach", in Proceedings of IEEE Global Telecommunications Conference (Globecom), Dallas, TX, November 2004.

[23] M. Felegyhazi and J. P. Hubaux, "Game theory in wireless networks: a tutorial", EPFL technical report, LCA-REPORT-2006-002, February, 2006.

[24] X. Qiu and K. Chawla, "On the performance of adaptive modulation in cellular systems," IEEE Transactions on Commununications, vol.47, no.6, pp.884-895, June 1999.

[25] S. T. Chung and A. J. Goldsmith, "Degrees of freedom in adaptive modulation: a unified view", IEEE Transactions on Commununications , vol.49, pp.1561-1571, September 2001.

[26] M. S. Barzaraa, Nonlinear programming: theory and algorithms, 2nd edition, John Wiley \& Sons, 1993.

[27] S. Boyd and L. Vandenberghe, Convex optimization, Cambridge University Press, 2004.

[28] R. Yates, "A framework for uplink power control in cellular radio systems", IEEE Journals on Selected Areas on Commununications, vol.13, no.7, pp.1341-1348, September 1995.

[29] G. J. Foschini and Z. Miljanic. "A simple distributed autonomous power control algorithm and its convergence", IEEE Transactions on Vehicular Technology, vol.42, no.4, pp.641-646, November 1993.

[30] G. Golub, Matrix computations, third edition, Johns Hopkins University Press, 1996.

[31] R. Jantti and S. L. Kim, "Second-order power control with asymptotically fast convergence," IEEE Journal on Selected Areas in Communication: Wireless Communication Series, vol. SAC-18 (3), pp.447-457, 2000

[32] J. J. Beek, P. O. Borjesson M. Boucheret, D. Landstrom, J. M. Arenas, P. Odling, C. Ostberg, M. Wahlqvist, and S. K. Wilson, "A time and frequency synchronization scheme for multiuser OFDM", IEEE Journals on Selected Areas on Commununications vol.17, no.11, pp.1900-1914, November 1999.

[33] S. Martello and P. Toth, Knapsack problems: algorithms and computer implementations, Wiley, West Sussex, England, 1990. 


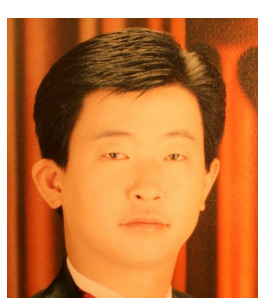

Zhu Han (S'01-M'04) received the B.S. degree in electronic engineering from Tsinghua University, in 1997, and the M.S. and Ph.D. degrees in electrical engineering from the University of Maryland, College Park, in 1999 and 2003, respectively.

From 2000 to 2002 , he is an R\&D Engineer of ACTERNA, Germantown, Maryland. From 2002 to 2003, he was a Graduate Research Assistant at the University of Maryland. From 2003 to 2006, he was a Research Associate at the University of Maryland. Currently, he is an assistant Professor in Electrical and Computer Engineering Department at Boise State University, Idaho, USA. His research interests include wireless resource allocation and management, wireless communications and networking, game theory, and wireless multimedia.

Dr. Han is Technical Program Co-Chair for IEEE Wireless Communication and Networking Conference 2007 and guest editor for special issue on crosslayer optimized wireless multimedia communistions, journal of advances in multimedia. He is a member of the Technical Programming Committee for the IEEE International Conference on Communications, the IEEE Vehicular Technology Conference, the IEEE Consumer Communications and Networking Conference, the IEEE Wireless Communications and Networking Conference, and the IEEE Globe Communication Conference.

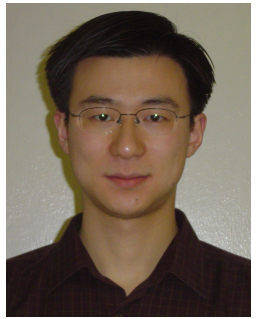

Zhu Ji received a Ph.D. degree in Electrical and Computer Engineering from University of Maryland, College Park in May 2007. He received B.S. and M.S. degrees in Electronic Engineering from Tsinghua University, Beijing, China, in 2000 and 2003, respectively. He is currently with Qualcomm, San Diego, CA. From 2003 to 2007, he was a graduate research assistant in the Communication and Signal Processing Laboratory, University of Maryland, College Park. From 2000 to 2002, he was a visiting student (research intern) in the Wireless and Networking Group at Microsoft Research Asia, Beijing, China. His research interests are in wireless communications and networking.

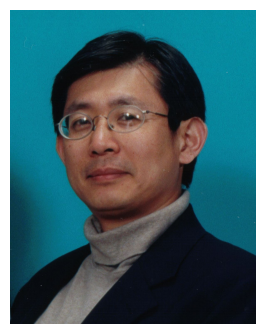

K. J. Ray Liu (F'03) is Professor and Associate Chair, Graduate Studies and Research, of Electrical and Computer Engineering Department, University of Maryland, College Park. His research contributions encompass broad aspects of wireless communications and networking, information forensics and security, multimedia communications and signal processing, bioinformatics and biomedical imaging, and signal processing algorithms and architectures.

Dr. Liu is the recipient of numerous honors and awards including best paper awards from IEEE Signal Processing Society (twice), IEEE Vehicular Technology Society, and EURASIP; IEEE Signal Processing Society Distinguished Lecturer, EURASIP Meritorious Service Award, and National Science Foundation Young Investigator Award. He also received various teaching and research recognitions from University of Maryland including university-level Distinguished ScholarTeacher Award and Invention of the Year Award, and college-level Poole and Kent Company Senior Faculty Teaching Award.

Dr. Liu is Vice President - Publications and on the Board of Governor of IEEE Signal Processing Society. He was the Editor-in-Chief of IEEE Signal Processing Magazine and the founding Editor-in-Chief of EURASIP Journal on Applied Signal Processing. 